\title{
Methodology for the analysis of a thermo-mechanically deformed optical system
}

Simone Nordera, Paolo Chioetto, Paola Zuppella, Emanuele Pace, Gianluca Morgante, et al.

Simone Nordera, Paolo Chioetto, Paola Zuppella, Emanuele Pace, Gianluca Morgante, Andrea Tozzi, Ciro del Vecchio, Antonio Scippa, Giuseppina Micela, Vania Da Deppo, "Methodology for the analysis of a thermo-mechanically deformed optical system," Proc. SPIE 11852, International Conference on Space Optics - ICSO 2020, 1185250 (11 June 2021); doi: $10.1117 / 12.2599824$

SPIE Event: International Conference on Space Optics - ICSO 2021, 2021, Online Only 


\section{International Conference on Space Optics-ICSO 2020}

Virtual Conference

30 March-2 April 2021

Edited by Bruno Cugny, Zoran Sodnik, and Nikos Karafolas
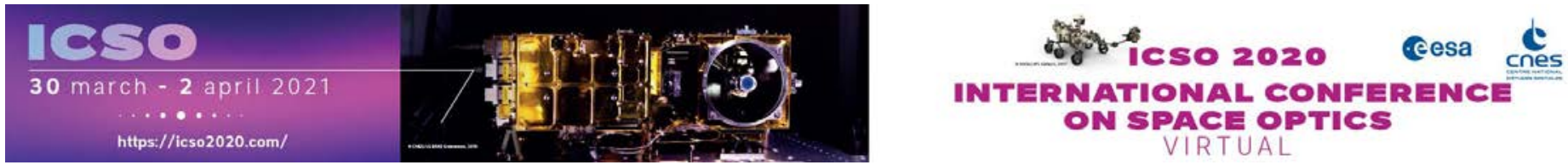

\section{Methodology for the analysis of a thermo-mechanically deformed optical system}

\section{Cesa issopereatings denes}




\title{
Methodology for the analysis of a thermo-mechanically deformed optical system
}

\author{
Simone Nordera*a,b , Paolo Chioetto ${ }^{\mathrm{a}, \mathrm{b}, \mathrm{c}}$, Paola Zuppella ${ }^{\mathrm{a}, \mathrm{b}}$, Emanuele Pace $^{\mathrm{d}}$, Gianluca Morgante , $^{\mathrm{e}}$ \\ Andrea Tozzi ${ }^{\mathrm{f}}$, Ciro Del Vecchio ${ }^{\mathrm{f}}$, Antonio Scippa ${ }^{\mathrm{g}}$, Giuseppina Micela ${ }^{\mathrm{h}}$, Vania Da Deppo ${ }^{\mathrm{a}, \mathrm{b}}$

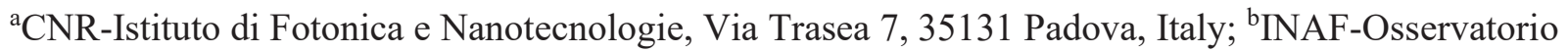 \\ Astronomico di Padova, Vicolo dell'Osservatorio 5, 35122 Padova, Italy; 'CISAS “G. Colombo", \\ Università di Padova, Via Venezia 15, 35131 Padova, Italy; 'Dipartimento di Fisica ed Astronomia,

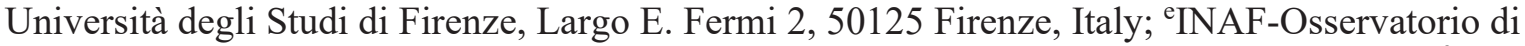 \\ Astrofisica e Scienza dello Spazio di Bologna, Via Piero Gobetti 93/3, 40129 Bologna, Italy; ${ }^{\text {INAF- }}$ \\ Osservatorio Astrofisico di Arcetri, Largo E. Fermi 5, 50125 Firenze, Italy; ${ }^{g}$ Dipartimento di \\ Ingegneria Industriale, Università degli Studi di Firenze, via S. Marta 3, 50139 Firenze, Italy; \\ ${ }^{h}$ INAF-Osservatorio Astronomico di Palermo, Piazza del Parlamento 1, 90134 Palermo, Italy
}

\begin{abstract}
The performance of as-built optical instruments strongly depends on thermal and structural loads, since these boundary conditions can affect the geometry of optical surfaces. Variations of temperature influence the volume, and the shape, of the structure proportionally to the coefficient of thermal expansion of the material, while mechanical loads, like gravity, may induce deformations on the optical elements according to the set of applied constraints. Those effects can introduce aberrations that degrade the performance of the optical system.

Since software for optical and thermo-structural analysis are usually different, a coupling methodology between these two fields of physics is needed. This is a step-by-step procedure through many platforms.

In this work, the procedure devised and used by the authors will be presented. At first, a thermo-mechanical analysis (depending on the loads involved) has to be performed, in order to obtain the final deformed geometry of the optical structure; COMSOL Multiphysics is the finite element solver (FEM) used for these analyses. Then an output data file, containing the coordinates of points belonging to the optical surface, can be generated. The output data are elaborated by a MATLAB routine that allows to convert the set of points into an $n$-th polynomial expression that best fits the surface data. The fitted polynomial surface is hence imported in ZEMAX ray-tracing software to study the optical performances of the system and the effects of thermo-mechanical loads.
\end{abstract}

Keywords: space camera, optics, thermoelastic analysis, FEM, stop analysis, polynomial fitting

\section{INTRODUCTION}

Before flying, a space optical instrument must successfully pass a series of ground qualification tests to be considered suitable for the space mission. The architecture design of the optical system must guarantee the continuous fulfilment of the requirements; this is checked through ray-tracing analysis and experimental tests, but these studies are often not sufficient to ensure good performances in every flight condition. Indeed, optical performance changes with the load conditions acting on a non-rigid body, i.e. an elastic material device.

Main loads that take place in space are of mechanical or thermal nature. Mechanical loads have a direct impact on the geometry of an elastic body, and they can be predominantly inertial or external (excluding inner mechanisms or contact phenomena). The difference between the presence of the gravity on-ground and its absence in space is the most common force that has to be considered during a space mission, while external loads can arise in different forms, discrete or distributed, instantaneous, constant or time dependent. For instance, a payload approaching a comet is subjected to dust jet flows coming from the nucleus as external load [1].

*simone.nordera@pd.ifn.cnr.it; phone +39-3454433122 
Instead, thermal loads result from heat transfer between the body and the external setting. Conduction, convection and radiation are the main three heat transfer methods, but convection is zero in an air-free setting and conduction only depends on the mechanical linkage with the rest of the structure. Radiation is a heat source that can strongly vary the spacecraft's temperature, and it is the main cause of heat dissipation in space, potentially leading the payload to cryogenic temperatures.

\section{STOP ANALYSIS}

The coupling of the structural and thermal behaviour of the instrument is solved through a finite element analysis (FEM), and the results must satisfy the structural and thermal requirements. Moreover, the effects that these strains have on the optical system must be predicted. This is the overall background of the so-called Structural-Thermal-Optical Performance analysis (STOP analysis). The chart in Figure 1 sums up the linkage among these three fields of physics and the sources of deformation as inputs to the geometrical optics analysis. However, variation of the optical performance may be induced not only by geometrical deformations, but also by optical parameters dependent on the stress state or temperature, like the refractive index [2].

Currently, different software packages are necessary to study this multi-physics linkage: thermo-structural analyses are performed in a FEM solver, while optical performances are obtained by means of a ray-tracing software. A correlation strategy between these two solvers has to be determined. The easiest method could be to export the result file from the FEM solver and to import it in the optical design program without any processing; however, the export and import file formats of these software are usually different. For instance, COMSOL Multiphysics FEM solver allows to export a result file as a spreadsheet of coordinates and displacements (and other, according to the desired physical properties) of mesh dependent points [3], while ZEMAX ray-tracing program sets up an optical surface through many surface types, which cannot be directly defined by such a spreadsheet. A post-processing step is necessary to convert the FEM output file to a file or expression importable in the optical software.

As the optical instrument may be subjected to different load cases, this STOP analysis must be performed for every load condition and, especially, for the most critical scenario. Besides, the post-processing step and the following ray-tracing analysis might be done also for the initial unloaded case as comparison with the optical design system, in order to check the equality of the results.

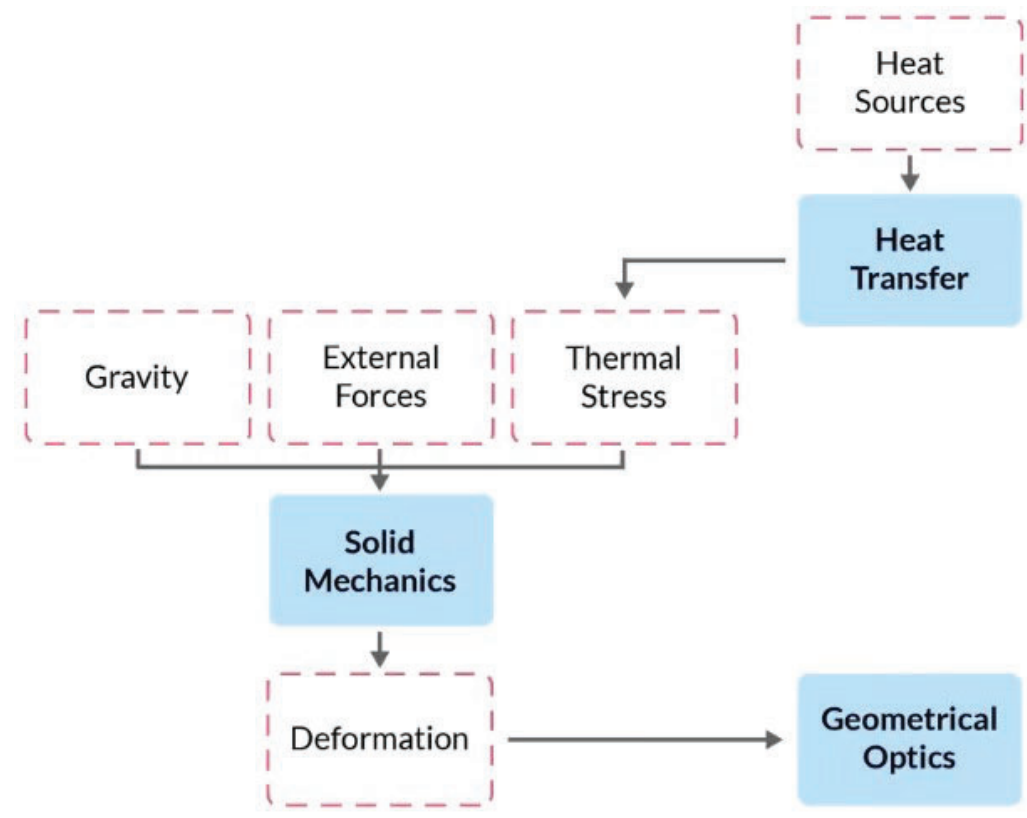

Figure 1. Summary chart of the multi-physics connections in a STOP (Structural-Thermal-Optical Performance) analysis. 


\section{MODELLING OF A THERMO-STRUCTURAL FEM ANALYSIS}

Basically, in a FEM model, a series of steps is set up: geometry, materials, properties, mesh, fields of physics, boundary conditions and type of study. A general model that can be suitable for every space optical device cannot be defined, because their applications and purposes, as their shapes and load conditions, are unlimited. The geometry and the materials of the instrument are such as to guarantee not only the required optical performance, but also the needed stiffness along the load direction. This implies the choice of structural materials like aluminium, titanium or silicon carbide, frequently used in space applications. For optics, deflection is a parameter often more critical than strength, especially for mirrors, quite sensitive to deformation errors [4]. In addition to stiffness, other parameters are important for the materials' choice, like density, thermal conductivity and thermal expansion.

\subsection{Coefficients of thermal expansion}

In a thermo-structural FEM analysis, while setting up many materials' parameters is usually not hard if the material is well known, much attention must be paid to those parameters which depend on temperature or stress. A material parameter that may change a lot especially for metals is the coefficient of thermal expansion (CTE); a good knowledge and control of this term is crucial for a thermo-structural analysis, as it links these two fields of physics. Scientific studies and experiments show that aluminium CTE, that is about $23 \cdot 10^{-6} \mathrm{~K}^{-1}$ at room temperature $\left(20^{\circ} \mathrm{C}\right)$, drastically drops at cryogenic temperatures $(<50 \mathrm{~K})$. If the CTE dependence on temperature and the temperature variation at which the material is subjected are known, two strategies may be carried out to perform the analysis: setting an average CTE or an instantaneous CTE [5].

The average CTE, also known as secant, can be determined through the integration of the coefficient of thermal expansion over the temperature range, divided by the temperature difference. Instead, the instantaneous CTE (tangent) is the slope of the thermal strain curve at a given temperature; it may be obtained through an interpolation method from known discrete points (Figure 2). The secant and tangent formulations are respectively:

$$
\begin{gathered}
\frac{\Delta L}{L_{0}}=\alpha\left(T, T_{0}\right) \Delta T=\alpha\left(T, T_{0}\right)\left(T-T_{0}\right) \\
\frac{d L}{L}=\alpha_{t}(T) d T
\end{gathered}
$$

Where $L_{0}$ is the initial length at the reference temperature $T_{0}, \alpha\left(T, T_{0}\right)$ is the secant thermal expansion coefficient and $\alpha_{t}(T)$ is the tangential thermal expansion coefficient, which is uniquely defined at each temperature.

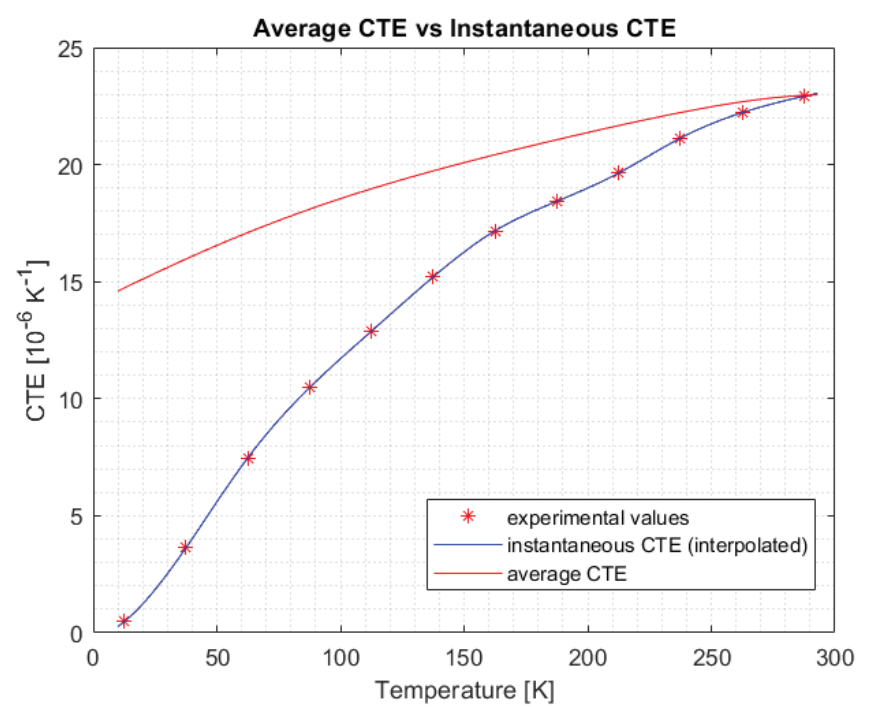

Figure 2. Experimental values and curves of instantaneous (interpolated) CTE and average CTE of aluminum 6061 T651. The discrete points are taken from the NASA Technical Memorandum [6]. 
The adopted strategy depends on the features offered by the software and the load conditions. If the final temperature of the body is not constant and a temperature gradient takes place, the average CTE changes along the surface, according to the final temperature of every surface point. In this case, tangent CTE is the recommended method.

\subsection{Coupling with thermal interface}

Regardless of the heat transfer method (conduction, convection or radiation), the resulting temperature variation leads to structural deformations as effect of the material thermal expansion, affecting the volume and the shape of the optical instrument in space. The presence of temperature gradients, different materials or mechanical constraints may lead to a no longer stress-free state. These thermal stresses cause differential deformations that distort the optical system, affecting its performance.

In order to reduce this effect, temperature gradients must be minimized and materials must be carefully chosen. Since a homogeneous variation of temperature in a single material causes a proportional expansion or contraction of the body, an optical structure consisting of a series of mirrors and supports, all made by the same material, only needs a refocusing mechanism to compensate for residual deformations [7]. A further solution is the use of low expansion materials; quartz glass is a common material for lenses and mirror, e.g. ULE® fused silica and ZERODUR ${ }^{\circledR}$ glass-ceramic show a nearzero coefficient of thermal expansion at room temperature, and for the optical elements mountings materials with very low CTE, such as Invar or $\mathrm{SiC}$, can be used [4]. Otherwise, the thermal compatibility of different materials may be employed; for instance, in Comet Interceptor ESA mission, the structural casing of the EnVisS optical head is made of a titanium alloy, because of its thermal compatibility with res[8]:-3], even if its density is much higher than aluminium.

\subsection{Meshing choice criteria}

In a thermo-mechanical analysis, the density of the mesh is always determined by a trade-off between computational times and reliability of the results. For an optical device, a finer mesh is recommended where a better knowledge of the results is required, if it can help the convergence of the FEM analysis or if it is demanded by the post-processing optical analysis. For example, contact phenomena require a very fine mesh on the source and destination surfaces of the contacting components, while a finer mesh in a compliant mechanism allows to reproduce more reliably the structural bending.

If an optical component like a concave mirror in a gravity field (with the optical axis perpendicular to the gravity vector) is considered, the structural analysis will return a deformed optical surface. According to the mesh shape and density of this surface, the post-processing ray-tracing analysis might lead to different optical performances. While the automatic mesh generator can lead to a non-uniform mesh, finer at the edges, a regular mesh may lead to more reliable results. The reason is that, in an optical design program (like ZEMAX), surfaces are created over a regular grid of points (64x64, $128 \times 128$, etc.); therefore, a regular mesh on the optical surface ensures a lower correlation error from FEM results to raytracing analysis. Indeed, the root-mean-square (RMS) value of the displacements in the optical axis direction changes with the mesh density. In a non-uniform mesh, finer areas affect more strongly the RMS displacement, because of the bigger number of data points; this distorted result is not comparable to the RMS value for a surface discretized by the regular grid of the optical software.

\section{FEM ANALYSIS RESULTS POST-PROCESSING}

Before developing a file converting strategy, it is necessary to find the best importing methodology in the optical software. In ZEMAX, for our present study an Extended Polynomial surface type has been considered suitable to be used. This choice allows to create an optical surface through a series of polynomial coefficients and a normalization radius. The surface sag is determined over every point of a regular grid in the $\mathrm{x}-\mathrm{y}$ plane; the values of $\mathrm{x}$ and $\mathrm{y}$ are divided by the normalization radius so to be dimensionless [9].

\subsection{Deformed optical surface polynomial fitting}

From the FEM solver, the deformed geometry can be exported as a .dat or .txt file with the displacements of the mesh nodes in the three axis directions. This file is processed by a MATLAB routine in order to obtain the coefficients of the polynomial expansion that best fits the imported surface. polyfitn, a MATLAB function written by John D'Errico, is an extension of the built-in polyfit function and it allows to perform fitting operations with more than one independent variable [10]. The input variables of this routine are the $\mathrm{x}$ and $\mathrm{y}$ coordinates, followed by the $\mathrm{z}$ coordinates, if the reference plane 
is the $\mathrm{x}-\mathrm{y}$ plane and the $\mathrm{z}$ coordinate is the sag; in addition, the polynomial degree (n) must be specified. The MATLAB entry takes the form of:

$$
\text { polymodel = polyfitn }([X, Y], Z, n)
$$

The fitting method used by polyfitn is a linear least square regression. It returns a structure with several fields, among which the polynomial coefficients, the variable names and the RMS fitting error. If $\mathrm{n}$ is the polynomial order, the total number of coefficients can be obtained as:

$$
\text { number_coeff }=\sum_{1}^{n+1} i
$$

Further functions (by J. D’Errico) that can help to manipulate the polynomial results are polyn2sympoly and polyvaln. polyn2sympoly allows to visualize the polynomial expression as a well-ordered sum of terms, in which every coefficient is multiplied by its independent variable; polyvaln is an extension of the built-in polyval and it permits to evaluate the polynomial function over the input $\mathrm{x}$ and $\mathrm{y}$ coordinates. The difference between the $\mathrm{z}$ coordinates of the deformed surface and the polyvaln values shows the fitting error point-by-point; the RMS average of this difference is equal to the RMS value returned by the polyfitn structure. Their formulation is:

$$
\begin{aligned}
& \text { P_expr = polyn2sympoly }(\text { polymodel }) \\
& \text { z_p }=\text { polyvaln }(\text { polymodel, }[\mathrm{X}, \mathrm{Y}])
\end{aligned}
$$

\subsection{Best fit sphere calculation}

If the surface approximately shows a spherical shape, the determination of the best fit sphere may be a useful reference index of deformation. According to the load case considered, not only the radius of curvature can change, but also the centre of the sphere; for example, a change in the position of the sphere's centre and not in the radius of curvature might mean a rigid-body displacement or a pure tilt/tip.

In MATLAB, a sphere fitting can be performed using the sphereFit function, written by Alan Jenning [11]. Given a set of data points, it calculates the radius and the centre of the sphere through a least square approximation:

$$
\text { [Center,Radius] = sphereFit(data) }
$$

After a thermo-mechanical analysis in which the instrument reaches cryogenic temperatures, its volume will be lower and so its dimensions. A best fit sphere operation on the resulting surface will show a reduction of the radius of curvature. In first approximation, the variation of the sphere's radius can be calculated as [4]:

$$
\Delta R_{C}=R_{C} \alpha(T) \Delta T
$$

where $\mathrm{R}_{\mathrm{C}}$ is the radius of curvature, $\alpha(\mathrm{T})$ is the average $\mathrm{CTE}$ and $\Delta \mathrm{T}$ is the temperature variation. In an optical system, this condition leads to a defocus, but it can be compensated by means of a refocusing mechanism.

\section{AN APPLICATION EXAMPLE: THE ARIEL PTM}

In this section, an application of this methodology is presented. The optical component is the Pathfinder Telescope Mirror (PTM), i.e. the demonstrator of the primary mirror (M1) of ARIEL's telescope [7]. The PTM mirror has been provisioned by MediaLario srl. This is a concave mirror with a spherical shape and an elliptical footprint (Figure 3a), and with a lightening pattern on the backside [12]. It is made of aluminium $6061 \mathrm{~T} 651$, whose coefficient of thermal expansion is depicted in Figure 2. In the FEM simulation, the mirror lies vertically on two horizontal quartz cylinders (mechanically fixed), it is affected by gravity, and it is subjected to a temperature reduction when brought from ambient to cryogenic conditions $(<50 \mathrm{~K})[13]$. The mesh is finer on the contacting surfaces and regular on the optical surface. The simulations have been done considering the mounting and environmental constraints the PTM mirror has been subjected during the interferometric cryo-tests performed at the Centre Spatial de Liège (CSL).

Two finite element analyses are performed in COMSOL [14]: a structural stationary analysis with only gravity and a thermo-structural stationary analysis with both gravity and temperature variation. In addition to the deformations focused on the contact areas with cylinders, the gravity analysis shows a forward or backward rotation of the PTM body, according 
to the position of the supporting glass cylinders with respect to the mirror (Figure $3 \mathrm{~b}$ ). Because of the high temperature variation (approximately $260 \mathrm{~K}$ ) the high value of the average CTE of aluminium over the considered temperature range, i.e. approximately $1610^{-6} \mathrm{~K}^{-1}$, displacement results of the thermo-structural analysis are several orders of magnitude bigger than those of the structural analysis, and volume contraction is the main phenomenon.

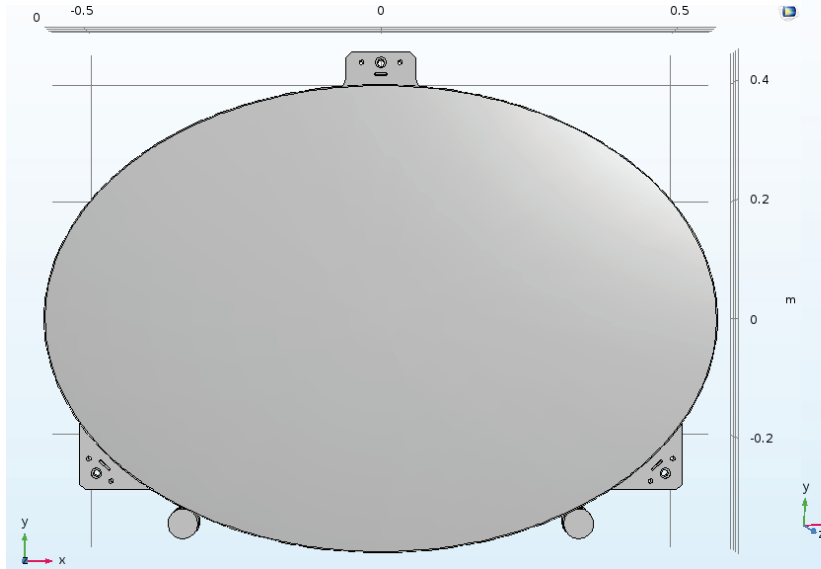

(a)

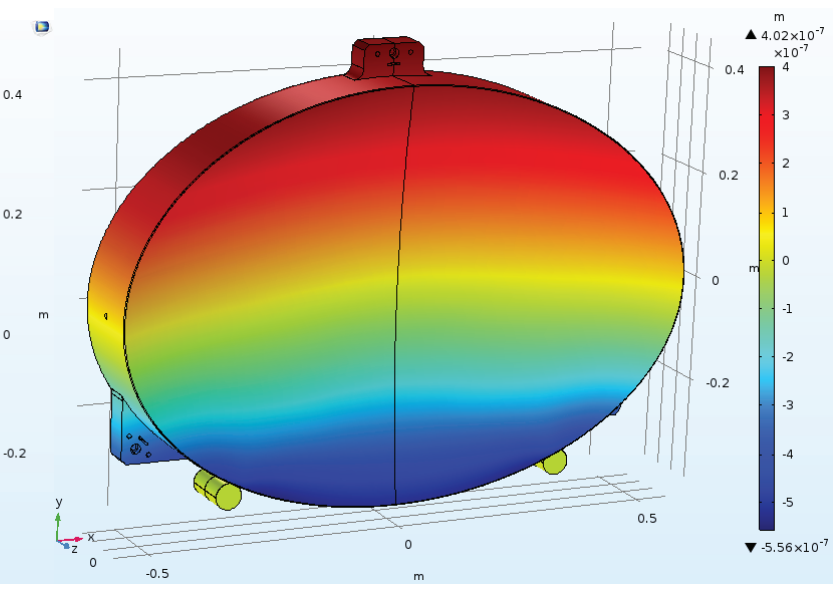

(b)

Figure 3. In (a), the PTM geometry over the cylinders. In (b), the displacement distribution along the direction of the optical axis (z axis), after the structural analysis in COMSOL.

After exporting the displacement results of the mirror surface obtained from COMSOL, the surface fitting is performed in MATLAB. The polynomial order is set as 6, because the corresponding RMS error is less than the measurement precision during manufacturing. The resulting coefficients are 28. The best fit sphere calculation returns the values of radius of curvature reported in Table 1 for 3 different cases: for the theoretically designed mirror surface (unloaded condition), the surface after structural (gravity) and thermo-structural (gravity and temperature variation) analysis.

Table 1. Best fit radius values of the unloaded PTM surface and after structural and thermo-structural analysis.

\begin{tabular}{|l|l|}
\hline Load Condition & Best Fit Radius [mm] \\
\hline Unloaded Condition & 2400.725 \\
\hline Gravity & 2400.724 \\
\hline Gravity \& Temperature Variation & 2390.934 \\
\hline
\end{tabular}

After the thermo-structural analysis, the variation of the best fit radius is $9.791 \mathrm{~mm}$. The analytical formula (8) returns a variation of curvature radius of $9.808 \mathrm{~mm}$, considering a reference temperature of $293 \mathrm{~K}$ and an average final temperature slightly lower than $36 \mathrm{~K}$. This formula may be valid for first approximation considerations, because the gravity deformation is negligible compared to the thermal contraction.

In ZEMAX, the polynomial coefficients are imported into the Extended Polynomial surface type, and the normalization radius is set as 1 . So, ray-tracing analyses can be performed to determine the optical performances of the mirror in each load condition. The mirror deformed by gravity is studied as inserted in an optical system that is intended to reproduce the interferometric analysis set-up used at the CSL, in which a spherical wavefront produced by an interferometric setup is reflected by the mirror. This analysis shows that tilt is the main aberration component (Figure 4), as it could be also deduced looking at the COMSOL results (Figure 3b). In the cryogenic case, instead, the curvature of the mirror is changing a lot from the original one and thus a high focus error is present if a refocusing of the interferometric set-up is not considered. 


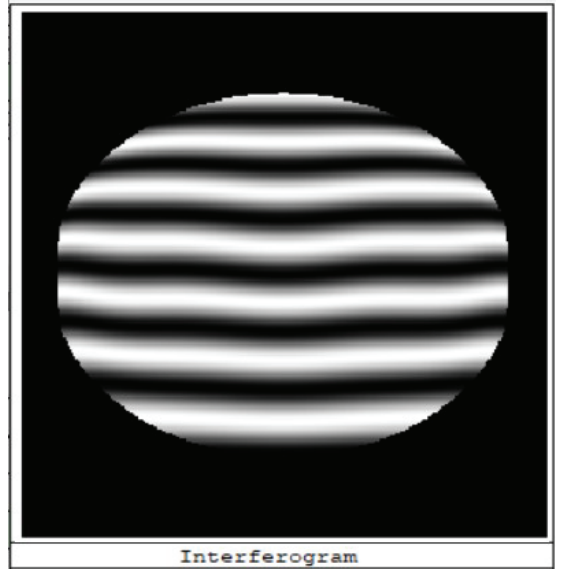

(a)

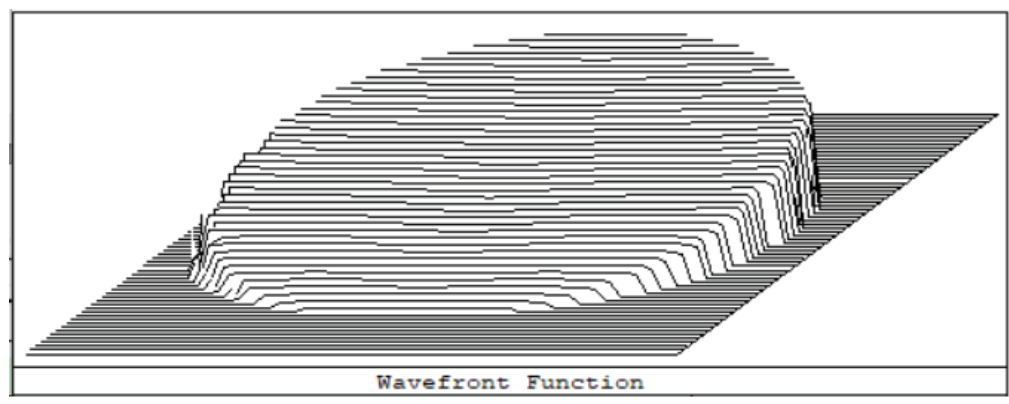

(b)

Figure 4. In (a) "Interferogram" and in (b) "Wavefront Map" simulated with ZEMAX applied to the PTM mirror surface deformed by gravity and rebuilt through polynomial coefficients. They show tilt as the main deformation component.

\section{CONCLUSIONS}

A good definition of the FEM model is a key step to predict the deformed shape of the instrument optical surfaces, which can be processed and analysed in a ray-tracing program. The surface's polynomial fitting is the recommended strategy, because it generates a series of coefficients which can be easily imported in the optical design software, so that these surfaces can be studied.

An application of this method consists of the STOP analysis of the ARIEL PTM, i.e. the optical analysis of the thermomechanically deformed demonstrator of the ARIEL primary mirror. After performing the FEM analysis in COMSOL, the deformed mirror's surface is fitted through the MATLAB routine and the polynomial coefficients are defined for the Zemax ${ }^{\circledR}$ Extended Polynomial surface type. Aberration plots show that the rebuilt optical surface is comparable with the output deformed surface in the FEM solver, therefore verifying the robustness of the methodology.

An in-depth optical analysis of these deformed surfaces is in progress, especially about the mirror at cryogenic temperatures, as it implies a refocusing adjustment of the optical system.

\section{ACKNOWLEDGMENTS}

This activity has been conducted and funded through some Agenzia Spaziale Italiana (ASI) contracts to the Istituto Nazionale di Astrofisica (INAF) (n. 2020-4-HH.0 and n. 2018-22-HH.0).

\section{REFERENCES}

[1] Jones, G. H., Snodgrass, C. and the Comet Interceptor Consortium, "Comet Interceptor: A Mission to a Dynamically New Solar System Object" (2019).

[2] Waxler, R. M., Cleek, G. W., "The Effect of Temperature and Pressure on the Refractive Index of Some Oxide Glasses", Journal of Research of the National Bureau of Standards. Section A, Physics and Chemistry, 77A(6): 755-763, (1973).

[3] "COMSOL Multiphysics Reference Manual, 5.6”, 1692-1694, (2020).

[4] Pepi, J. W., [Opto-Structural Analysis], SPIE Press, 39-44, 89, 106-110, (2018).

[5] "Structural Mechanics Module, User's Guide, COMSOL 5.6", 238-247, (2020).

[6] Horton, J. C., Smith, C. F., Ruff, R. C., "NASA Technical Memorandum, Low Temperature Thermal Expansion of Structural Metals", NASA TM X-53436, (1966). 
[7] Da Deppo, V., et al., "An afocal telescope configuration for the ESA ARIEL mission”, CEAS Space J, 9:379398, Springer, (2017).

[8] Jones, G., Cole, R., “EnVisS Instrument Description”, MSSL-ENVISS-RP-19001 Issue B, (2019).

[9] “Zemax ${ }^{\circledR}$ 13, Optical Design Program, User’s Manual”, 340-341, 2014.

[10] John D'Errico (2021). polyfitn (https://www.mathworks.com/matlabcentral/fileexchange/34765-polyfitn), MATLAB Central File Exchange. Retrieved February 8, 2021.

[11] Alan Jennings (2021). Sphere Fit (least squared) (https://www.mathworks.com/matlabcentral/fileexchange /34129-sphere-fit-least-squared), MATLAB Central File Exchange. Retrieved February 8, 2021.

[12] Terraneo, M., "PTM Thermoelastic analysis, COM-SP063 - PTM Mirror", ARI-ML-TN-001, (2019).

[13] Roose, S., "ARIEL PTM WFE measurements during cryo-optical test", RP-CSL-APTM-20020, issue 2.1, (2020).

[14] Nordera, S., "Cryotesting of ARIEL M1 mirror and coating process qualification for derisking ARIEL Schedule, Thermo-structural static finite element analysis of the PTM setup for cryotesting", INAF-CSL-PTM-RP-004, (2020). 